\title{
Prognostic Value of Peak Oxygen Uptake in Patients Supported With Left Ventricular Assist Devices (PRO-VAD)
}

Kiran K. Mirza, MD, ${ }^{\mathrm{a}}$ Mariusz K. Szymanski, MD, PhD, ${ }^{\mathrm{b}}$ Thomas Schmidt, PhD, MA, ${ }^{\mathrm{c}}$ Nicolaas de Jonge, MD, PhD, Darshan H. Brahmbhatt, MA, MB, BCHIR, ${ }^{\mathrm{d}, \mathrm{e}}$ Filio Billia, MD, PHD, ${ }^{\mathrm{d}}$ Steven Hsu, MD, ${ }^{\mathrm{f}}$ Guy A. MacGowan, MD, ${ }^{\mathrm{g}, \mathrm{h}}$ Djordje G. Jakovljevic, PHD, ${ }^{,, h, i}$ Piergiuseppe Agostoni, MD, PHD, ${ }^{\mathrm{j}, \mathrm{k}}$ Filippo Trombara, MD, ${ }^{1}$ Ulrich Jorde, MD, PHD,

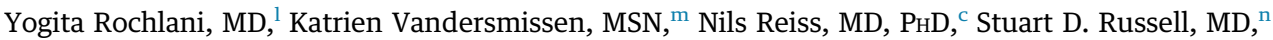
Bart Meyns, MD, PHD, ${ }^{\mathrm{m}}$ Finn Gustafsson, MD, PHD, DMSc, ${ }^{\mathrm{a}, \mathrm{o}}$ on behalf of the PRO-VAD investigators

\section{ABSTRACT}

OBJECTIVES The purpose of this study was to examine whether peak oxygen uptake $\left(\mathrm{pVO}_{2}\right)$ and other cardiopulmonary exercise test (CPET)-derived variables could predict intermediate-term mortality in stable continuous flow LVAD recipients.

BACKGROUND $\mathrm{pVO}_{2}$ is a cornerstone in the selection of patients for heart transplantation, but the prognostic power of $\mathrm{pVO}_{2}$ obtained in patients treated with a left ventricular assist device (LVAD) is unknown.

METHODS We collected data for $\mathrm{pVO}_{2}$ and outcomes in adult LVAD recipients in a retrospective, multicenter study and evaluated cutoff values for $\mathrm{pVO}_{2}$ including: 1) values above or below medians; 2) grouping patients in tertiles; and 3) $\mathrm{pVO}_{2} \leq 14 \mathrm{ml} / \mathrm{kg} / \mathrm{min}$ if the patient was not treated with beta-blockers (BB) or $\mathrm{pVO}_{2} \leq 12 \mathrm{ml} / \mathrm{kg} / \mathrm{min}$ if the patient was taking BB therapy.

RESULTS Nine centers contributed data from 450 patients. Patients were $53 \pm 13$ years of age; $78 \%$ were male; body mass index was $25 \pm 5 \mathrm{~kg} / \mathrm{m}^{2}$ with few comorbidities (stroke: $11 \%$; diabetes: $18 \%$; and peripheral artery disease: $4 \%$ ). The cause of heart failure (HF) was most often nonischemic (66\%). Devices included were the HeartMate II and 3 (Abbott); and Heartware ventricular assist devices Jarvik and Duraheart (Medtronic). The index CPET was performed at a median of 189 days (154 days-225 days) after LVAD implantation, and mean $\mathrm{pVO}_{2}$ was $14.1 \pm 5 \mathrm{ml} / \mathrm{kg} / \mathrm{min}(47 \% \pm 14 \%$ of predicted value). Lower $\mathrm{pVO}_{2}$ values were strongly associated with poorer survival regardless of whether patients were analyzed for absolute $\mathrm{pVO}_{2}$ in $\mathrm{ml} / \mathrm{kg} / \mathrm{min}, \mathrm{pVO}_{2} \leq 12 \mathrm{BB} / 14 \mathrm{ml} / \mathrm{kg} / \mathrm{min}$, or as a percentage of predicted $\mathrm{pVO}_{2}$ values $(P \leq 0.001$ for all). For patients with $\mathrm{pVO}_{2}>12 \mathrm{BB} / 14$ and ventilation/carbon dioxide relationship $\left(\mathrm{VE} / \mathrm{VCO}_{2}\right)$ slope $<35$, the 1-year survival was $100 \%$.

CONCLUSIONS Even after LVAD implantation, $\mathrm{pVO}_{2}$ has prognostic value, similar to HF patients not supported by mechanical circulatory support devices. (PROgnostic Value of Exercise Capacity Measured as Peak Oxygen Uptake $\left[\mathrm{pVO}_{2}\right]$ in Recipients of Left Ventricular Assist Devices [PRO-VAD]; NCTO4423562) (J Am Coll Cardiol HF 2021; $\left.: \mathbf{\square - \square}\right)$ (c) 2021 by the American College of Cardiology Foundation.

From the ${ }^{a}$ Department of Cardiology, Rigshospitalet, Copenhagen, Denmark; ${ }^{b}$ Heart Lung Center Utrecht, Utrecht, the Netherlands; 'Schüchtermann-Klinik Bad Rothenfelde, Institute for Cardiovascular Research, Bad Rothenfelde, Germany, and Institute for Cardiology and Sports Medicine, German Sports University Cologne, Cologne, Germany; ${ }^{\mathrm{d}}$ Peter Munk Cardiac Centre, Division of Cardiology, Ted Rogers Centre for Heart Research, University Health Network, University of Toronto, Toronto, Ontario,

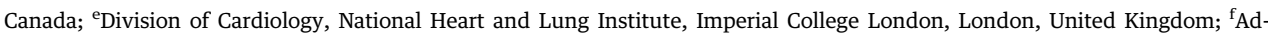
vanced Heart Failure, Mechanical Circulatory Support, Transplant Cardiology, Johns Hopkins Hospital, Baltimore, Maryland, USA; ${ }^{g}$ Department of Cardiology, Freeman Hospital and Biosciences Institute, Newcastle University, Newcastle upon Tyne, United Kingdom; hFaculty of Health and Life Sciences, Coventry University, University Hospital Coventry and Warwickshire, United Kingdom; ${ }^{\mathrm{i} D e p a r t m e n t}$ of Cardiology, Freeman Hospital and Biosciences Institute, Newcastle University, Newcastle upon Tyne, United Kingdom; ${ }^{j}$ Centro Cardiologico Monzino, Istituto di Ricovero e Cura a Carattere Scientifico (IRCCS), Milano, Italy; ${ }^{\mathrm{D} D e-}$ partment of Clinical Sciences and Community Health, Cardiovascular Section, University of Milano, Milano, Italy; ${ }^{\mathrm{l} M o n t e f i o r e}$ Einstein Center for Heart and Vascular Care New York, New York City, New York, USA; mepartment of Cardiac Surgery, Katholieke Universiteit Leuven, Leuven, Belgium; ${ }^{\mathrm{n}}$ Department of Cardiology, Duke University Health System, Durham, North Carolina, USA; and the ${ }^{\circ}$ Department of Clinical Medicine, Rigshospitalet, Copenhagen, Denmark. 


\section{ABBREVIATIONS AND ACRONYMS \\ $12 \mathrm{BB} / 14$ = peak oxygen uptake cutoff $(\mathrm{ml} / \mathrm{kg} / \mathrm{min})$ of 12 if taking beta-blocker therapy and 14 if not taking beta- blocker therapy \\ BB = beta-blocker \\ BTD/-T = bridge to decision/ transplantation \\ DT = destination therapy \\ $\mathrm{CO}=$ cardiac output \\ CPET = cardiopulmonary \\ exercise test \\ LVAD = left ventricular assist device \\ $\mathrm{PVO}_{2}=$ peak oxygen uptake \\ QOL = quality of life \\ $\mathrm{VE} / \mathrm{VCO}_{2}=$ ventilation/carbon}

dioxide relationship
S

urvival rates after implantation with a durable left ventricular assist device (LVAD) have greatly increased over the last decade, and improvements in LVAD technology have lowered the risk of adverse events (1-3). In fact, recent studies have shown that short-term and intermediate term survival after LVAD implantation parallels that of cardiac transplantation (4). Furthermore, most LVAD recipients experience significant improvement in heart failure (HF) symptoms and quality of life (QOL) (5-7).

Although the preferred treatment for advanced, refractory HF remains heart transplantation, waiting time for donor organs is increasing in most countries. In a recent report from the ELEVATE (European registry of patients implanted with a full magnetically levitated LVAD) registry (3) of Heartmate3 (Abbott Laboratories) recipients, $<10 \%$ underwent transplantation after 2 years of device support, despite the fact that most received implants as a bridge-to-transplantation (BTT) (8). In the United States, the new allocation system (9) prioritizes patients on temporary mechanical circulatory support (MCS), which will likely increase waiting time for stable LVAD recipients. This highlights the potential need for prioritization of stable BTT LVAD recipients for organ allocation or even for consideration of the indication for transplantation, by risk stratification similar to guidelines applied for elective patients with advanced HF not supported with MCS (10). A cornerstone in selection of patients for heart transplantation listing is measurement of peak oxygen uptake $\left(\mathrm{pVO}_{2}\right)$ by means of a cardiopulmonary exercise test (CPET). In stable HF patients, $\mathrm{pVO}_{2}$ has been shown to predict mortality with high accuracy (11), and other CPET-derived measurements such as ventilation/carbon dioxide relationship $\left(\mathrm{VE} / \mathrm{VCO}_{2}\right)$ slope (12) may provide additional information. Recently, $\mathrm{pVO}_{2}$ has been studied extensively in LVAD recipients and has been reported to remain reduced even in patients with significant symptomatic improvement (7). However, it is unknown whether $\mathrm{pVO}_{2}$ maintains its prognostic power in patients with $\mathrm{HF}$ once they are supported with an LVAD.
The present authors hypothesized that $\mathrm{pVO}_{2}$, as well as other CPET-derived variables, could predict mortality in stable recipients with a continuous flow LVAD, which could make the test potentially useful to select patients for cardiac transplantation listing also in this group of patients.

\section{METHODS}

A retrospective multicenter study was designed to collect data for $\mathrm{pVO}_{2}$ in LVAD recipients. Based on earlier published reports (described elsewhere [7]), 34 centers performing CPET in LVAD recipients, either as part of research projects or as standard of care, were identified and invited to participate in this study. Adult patients supported by LVAD with at least 1 postimplantation CPET (with available $\mathrm{pVO}_{2}$ ) were eligible for inclusion. All CPET protocols were accepted for inclusion regardless of initial workload and duration of workload steps used (stepwise increase every 2 or 3 minutes or continuous increase every second). Exclusion criteria included concomitant right ventricular assist devices and partially implanted LVADs. At the end of data collection (September 2020), 9 centers (Supplemental Table 1) had provided anonymized data to the database located at Rigshospitalet, Copenhagen, Denmark.

The following outcomes were collected: ongoing on-device, transplantation, explantation caused by cardiac recovery, pump exchange caused by thrombosis, and death. The primary endpoint was death, censoring for explantation for recovery and transplantation. Time to any endpoint was calculated as time elapsed from the first CPET performed after LVAD implantation (the index CPET) until the outcome of interest.

Beta-blocker dosages were reported in equivalents of metoprolol succinate. Doses were calculated as $100 \mathrm{mg}$ of metoprolol succinate, $\sim 5 \mathrm{mg}$ bisoprolol, $\sim 25 \mathrm{mg}$ of carvedilol, $\sim 200 \mathrm{mg}$ of labetalol, $\sim 80 \mathrm{mg}$ of propranolol, and $\sim 160 \mathrm{mg}$ of sotalol. Loop diuretic agents were reported in equivalent doses of furosemide. Doses were calculated as $40 \mathrm{mg}$ of furosemide; $\sim 20 \mathrm{mg}$ of torsemide, and $\sim 1 \mathrm{mg}$ of bumetanide. Mean arterial blood pressure (MAP) was calculated as diastolic blood pressure $+1 / 3 \times$ [systolic blood pressure - diastolic blood pressure].

The authors attest they are in compliance with human studies committees and animal welfare regulations of the authors' institutions and Food and Drug Administration guidelines, including patient consent where appropriate. For more information, visit the Author Center. 
Exercise tests were performed using protocols and LVAD settings as defined by the local center and staff. The LVAD settings were kept constant throughout the exercise test.

Percentage of predicted $\mathrm{pVO}_{2}$ (\% Pred $\mathrm{pVO}_{2}$ ) was calculated using the Hansen formula $(13,14)$. The contributing centers were asked to provide a $\mathrm{VE} / \mathrm{VCO}_{2}$ slope for CPET according to local methods of calculation, and if $\mathrm{VE} / \mathrm{VCO}_{2}$ was not used, the value was calculated by regression analysis (15) throughout the entire CPET (16), because we planned to use this value in case of suboptimal $\mathrm{pVO}_{2}$ (respiratory exchange ratio $[\mathrm{RER}]<1.05)$ in accordance with current criteria for transplant listing (10). Neither anaerobic threshold nor periodic breathing was included in the analyzes.

The study was conducted in accordance with the Declaration of Helsinki. Danish approval numbers were $\mathrm{H}-1-2012-092, \mathrm{H}-3-2013-010, \mathrm{H}-15 \mathrm{O}-21-598, \mathrm{H}-$ 17029488, 3-3013-1365/1, 3-3013-1365-2. The study was registered in the U.S. Clinical.trials.gov (NCT04423562).

\section{STATISTICS}

Continuous variables were expressed as mean \pm SD if parametric, as median and interquartile range (IQR) if nonparametric, and categorical variables were described with frequencies and percentages. Relationships between continuous variables were evaluated using Pearson's and Spearman's rank correlation coefficient as appropriate.

The impact of $\mathrm{pVO}_{2}$ on survival was assessed by use of the Kaplan-Meier method both for absolute $\mathrm{pVO}_{2}$ and \%Pred $\mathrm{pVO}_{2}$ data. The following cutoff values for $\mathrm{pVO}_{2}$ were tested for outcome analyses: 1 ) above or below median $\mathrm{pVO}_{2}$;2) grouping tertiles of $\mathrm{pVO}_{2}$; and 3) $\mathrm{pVO}_{2} \leq 14 \mathrm{ml} / \mathrm{kg} / \mathrm{min}$ if intolerant to beta-blockers and $\mathrm{pVO}_{2} \leq 12 \mathrm{ml} / \mathrm{kg} / \mathrm{min}$ if the patient was taking beta-blockers (10). Cases with missing values were excluded from the respective analyses, and no imputations were performed. Cox regression analysis was used to adjust for the impact of age, body mass index, and indication for therapy (ie, destination therapy [DT] vs. not DT) on the relationship between $\mathrm{pVO}_{2}$ and survival. To assess whether time from LVAD implantation to the time of the index CPET modified the predictive power of $\mathrm{pVO}_{2}$ on mortality, an interaction analysis was performed containing the interaction term $\mathrm{pVO}_{2}$ less than versus greater than the median (ie, $<l \geq$ median) $\mathrm{x}$ time from implantation to CPET performed earlier than versus later than the median time from implant (ie, $<\mid \geq$ median).

\begin{tabular}{|c|c|}
\hline Age, y & $53.0 \pm 13.0$ \\
\hline Females & $97.0(22)$ \\
\hline Whites & $399.0(89)$ \\
\hline Body mass index, $\mathrm{kg} / \mathrm{m}^{2}$ & $25.0 \pm 5$ \\
\hline Nonischemic cardiomyopathy & $292.0(66)$ \\
\hline \multicolumn{2}{|l|}{ Co-morbidity } \\
\hline Stroke & $46.0(11)$ \\
\hline Diabetes & $78.0(18)$ \\
\hline Peripheral artery disease & $18.0(4)$ \\
\hline LOS after LVAD implantation, days & $45.0 \pm 26$ \\
\hline Duration of HF prior to LVAD implantation, $y$ & $3.7(1-10)$ \\
\hline MAP, mm Hg & $78.0 \pm 11$ \\
\hline Resting $H R$, $\min ^{-1}$ & $88.0 \pm 18$ \\
\hline Cardiac implantable-defibrillator device & $279.0(64)$ \\
\hline Destination therapy & $102.0(23)$ \\
\hline \multicolumn{2}{|l|}{ Device } \\
\hline Heartmate II & $173.0(38)$ \\
\hline Heartmate 3 & $102.0(23)$ \\
\hline Heartware & $155.0(34)$ \\
\hline Jarvik & $18.0(4)$ \\
\hline Duraheart & $2.0(<1)$ \\
\hline Days from LVAD implantation to CPET & $189.0(154-225)$ \\
\hline \multicolumn{2}{|l|}{ Medications } \\
\hline Beta-blocker & $206.0(48)$ \\
\hline Metoprolol equivalent dose, mg & $68.7 \pm 64$ \\
\hline Loop diuretics & $248.0(58)$ \\
\hline Furosemide equivalent dose, $\mathrm{mg}$ & $60.0 \pm 69$ \\
\hline \multicolumn{2}{|l|}{ CPET variables } \\
\hline $\mathrm{PVO}_{2}, \mathrm{ml} / \mathrm{kg} / \mathrm{min}$ & $14.1 \pm 5.0$ \\
\hline Percentage of predicted peak oxygen uptake & $47.1 \pm 13.9$ \\
\hline Respiratory exchange ratio & $1.16 \pm 0.12$ \\
\hline $\mathrm{VE} / \mathrm{VCO}_{2}$ slope & $37.1(32.7-42.2)$ \\
\hline Test duration, $\mathrm{s}$ & $563.0 \pm 226$ \\
\hline Power, W & $89.0 \pm 38$ \\
\hline \multicolumn{2}{|c|}{$\begin{array}{l}\text { Values are mean } \pm S D, n(\%) \text { or interquartile range }(\text { IQR }) \text {, unless otherwise stated. } \\
C P E T=\text { cardiopulmonary exercise test; } C P E T=\text { cardiopulmonary exercise test; } \\
\mathrm{HF}=\text { heart failure; } \mathrm{LOS}=\text { length of (hospital) stay; } \mathrm{LVAD}=\text { left ventricular assist } \\
\text { device; } \mathrm{MAP}=\text { mean arterial blood pressure; } P V O_{2}=\text { peak oxygen uptake; } \\
\mathrm{VE} / \mathrm{VCO}_{2}=\text { ventilation } / \text { carbon dioxide relationship; } \mathrm{W}=\text { watts. }\end{array}$} \\
\hline
\end{tabular}

Statistical analyses were performed using SPSS software statistics (IBM) for Windows version 25.0 (Microsoft), and statistical significance was defined as a 2-tailed $P$ value $<0.05$. R Studio software version 1.3.1093 (R Foundation for Statistical Computing) was used for statistical presentation.

\section{RESULTS}

This study included 450 adult LVAD recipients with a postimplant CPET with $\mathrm{pVO}_{2}$. This corresponds to $41 \%$ of all patients who received implants during the data collection period in the participating centers (Supplemental Table 2). The index CPET was 
performed on average $251 \pm 259$ days after LVAD implantation (Table 1). The most commonly implanted devices were HeartMate II (Abbott) and Heartware ventricular assist device (HVAD) (Medtronic Inc). Patients were most often middle aged, White males (Table 1). Cause of HF was non-ischemic for most patients and peri-implant heart rhythm was most often sinus. More than one-half of the patients were implanted with pacing devices (64\%), of which most were implantable cardioverter-defibrillators (72\%). The burden of co-morbidities (eg, stroke, diabetes, or peripheral artery disease) was relatively low as shown in Table 1. Six-minute walk test was available from 87 patients, and the walking distance was $404 \pm 132 \mathrm{~m}$.

At the time of the CPET, only a little more than one-half of the patients received loop diuretic agents with an average dose of $60 \mathrm{mg}$ (furosemide equivalents), and beta-blockers were prescribed to fewer than one-half of the patients with an average dose of $69 \mathrm{mg}$ (metoprolol equivalents).

CARDIOPULMONARY EXERCISE TEST. Most tests were performed using an upright bicycle (93\%), followed by a test on a treadmill (6\%). Data were missing for 2 tests $(0.4 \%)$. Exercise capacity was severely reduced with an average $\mathrm{pVO}_{2}$ in the total cohort of $14.1 \pm 5 \mathrm{ml} / \mathrm{kg} / \mathrm{min}$, equivalent to $47 \%$ of the expected value. The average RER indicated a maximal or near maximal effort (average RER $=1.16$ ). Considerable variations were seen in test durations $(563 \pm 226 \mathrm{~s})$ and test intensities (maximal power achieved: $89 \pm 38$ W). A total of $49 \%$ of the patients had $\mathrm{pVO}_{2}$ below the accepted values for heart transplant listing (10). The $\mathrm{VE} / \mathrm{VCO}_{2}$ slope was missing from 50 (11\%) of the tests; median (IQR) $\mathrm{VE} / \mathrm{VCO}_{2}$ slope was 37.1 (32.7-42.0); 62\% had $\mathrm{VE} / \mathrm{VCO}_{2}$ slope $>35$.

SURVIVAL. After an average of $795 \pm 666$ days from index $\mathrm{pVO}_{2}$ to the end of follow-up, the following distribution of outcomes was observed: ongoing on device: 179 (40\%); transplantation: 169 (37.5\%); explantation: 16 (3.5\%); death: 86 (19\%). Comparing baseline characteristics of BTT patients who received transplants with those who were ongoing on device at the end of follow-up (excluding explanted patients), the authors found no significant differences in age, race, sex, body mass index, $\mathrm{HF}$ duration prior to LVAD implantation, presence of cardiac implantabledevices or comorbidities (stroke, diabetes, peripheral artery disease, data not shown).

Dividing patients into tertiles by peak $\mathrm{VO}_{2}$ clearly demonstrated that a lower $\mathrm{pVO}_{2}$ was significantly associated with poorer survival $(P<0.001)$ (Figure 1A). Similar results were found when patients were divided according to percentage of predicted $\mathrm{pVO}_{2}$ values $(P=0.001$ ) (Figure $1 \mathrm{~B})$.

After exclusion of 67 tests with RER $<1.05$, the findings remained statistically significant $(P=0.003)$. Upon excluding 105 patients with test duration $<300$ $\mathrm{s}$, the prognostic value of $\mathrm{pVO}_{2}$ remained unchanged $(P=0.001)$, and the same was true after excluding 139 patients with test durations of $<300$ or $>900 \mathrm{~s}$ $(P=0.001)$.

Using the accepted $\mathrm{pVO}_{2}$ threshold for transplantation listing of 12 (beta-blocker) or 14 (no betablocker) $\mathrm{ml} / \mathrm{kg} / \mathrm{min}$ also resulted in a significant and large difference in mortality (Figure $1 \mathrm{C}$ ). $\mathrm{VE} / \mathrm{VCO}_{2}$ slope values higher than the median were associated with lower survival rates $(P=0.023)$. Upon grouping patients into tertiles, this association became clearer $(P=0.001)$ (Figure 2A), and when dividing patients into subgroups according to the generally accepted $\mathrm{VE} / \mathrm{VCO}_{2}$ slope threshold of 35 (10), higher mortality in the higher $\mathrm{VE} / \mathrm{VCO}_{2}$ slope group was observed $(P<0.001)$ (Figure 2B). Combining $\mathrm{pVO}_{2}$ and $\mathrm{VE} / \mathrm{VCO}_{2}$ slope provided excellent discrimination of patients with very low risk. Grouping patients as: 1 ) $\mathrm{pVO}_{2}>12$ $\mathrm{BB} / 14 \mathrm{ml} / \mathrm{kg} / \mathrm{min}$ (no $\mathrm{BB}$ ) and $\mathrm{VE} / \mathrm{VCO}_{2}$ slope $\leq 35 ; 2$ ) $\mathrm{pVO}_{2} \leq 12 \mathrm{BB} / 14 \mathrm{ml} / \mathrm{kg} / \mathrm{min}$ (no $\mathrm{BB}$ ) and $\mathrm{VE} / \mathrm{VCO}_{2}$ slope $>35$; and 3 ) the remaining patients demonstrated $100 \%$ 1-year survival and close to $100 \%$ survival after more than 3 years of follow-up in group $1(P<0.0001)$ (Supplemental Figure 1$)$.

Excluding treadmill tests (6\%) did not change the prognostic impact of $\mathrm{pVO}_{2}$ grouping patients according to median, tertiles or when using the cutoffs of 12 $\mathrm{BB} / 14 \mathrm{ml} / \mathrm{kg} / \mathrm{min}$ with or without $\mathrm{VE} / \mathrm{VCO}_{2}$ slope $(P<0.001$ for all, graphs not shown).

To determine if the association between $\mathrm{pVO}_{2}$ and mortality was independent of patient age and anthropometric variables as well as device indication in Cox regression analysis age, sex, body mass index, and therapy indication (DT versus not DT) were adjusted for. Cox regression analysis confirmed a higher risk of death for patients with lower $\mathrm{pVO}_{2}$ (Table 2). Furthermore, interaction analysis revealed no significant differences in the predictive power of $\mathrm{pVO}_{2}$ on mortality for CPET performed early versus late (ie, before or after the median time to CPET) after LVAD implantation ( $p$ for interaction $=0.86$ ). No interaction with flow type (centrifugal/axial) on the association between $\mathrm{pVO}_{2}$ and survival was found ( $p$ for interaction $=0.39$ ).

A combined endpoint of death or HF hospitalization was also explored. The combined outcome was strongly predicted by lower $\mathrm{pVO}_{2}$ values $(P \leq$ 0.001 for all) (Figure 3, Supplemental Figure 2). 
FIGURE 1 Peak Oxygen Uptake

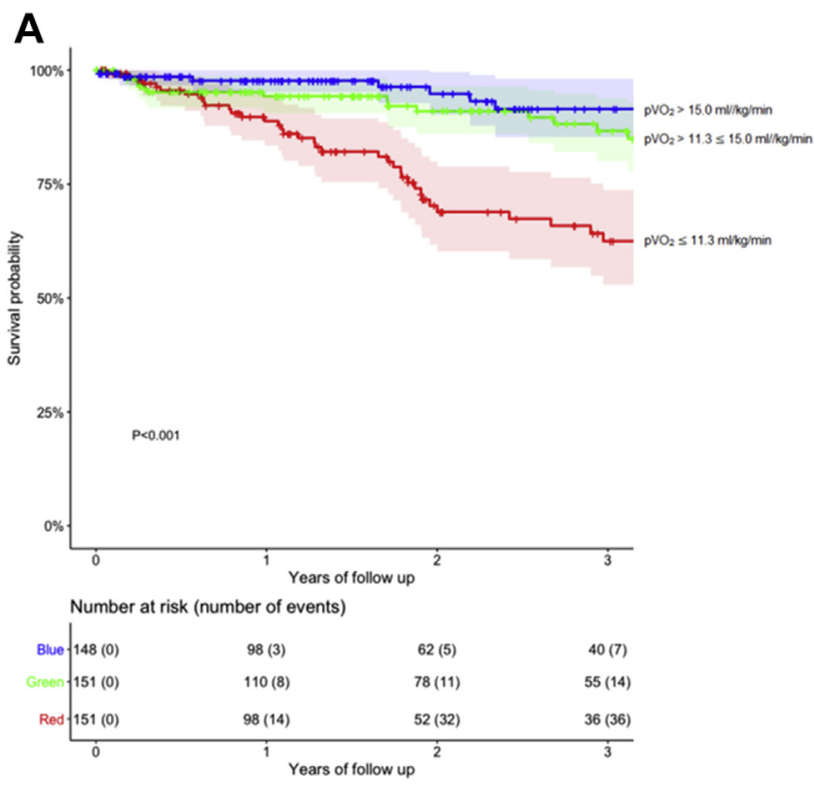

(A) Survival according to peak oxygen uptake $(\mathrm{ml} / \mathrm{kg} / \mathrm{min})$ in tertiles. $\mathrm{pVO}_{2}=$ survival according to peak oxygen uptake ( $\mathrm{pVO} \mathrm{O}_{2}$ in tertiles. (B) Kaplan-Meier plot shows survival according to the percentage of predicted peak oxygen uptake (\%Pred $\mathrm{pVO}_{2}$ ). Survival in patients with percentage of predicted peak oxygen uptake (\%Pred $\mathrm{pVO}_{2}$ ) less versus greater than the group median. (C) Kaplan-Meier plot shows survival according to peak oxygen uptake $(\mathrm{ml} / \mathrm{kg} / \mathrm{min})$ using the ISHLT cutoffs for evaluating transplant listing. ISHLT = International Society of Heart and Lung Transplantation.

Continued on the next page

Among the 289 patients still alive and ongoing on device 1 year after the index $\mathrm{pVO}_{2}$ test (ie, excluding transplanted, explanted and patients who died within 1 year after the index CPET), the crude incidence of $\mathrm{HF}$ hospitalizations was 0.14 per patient year (ppy) in patients with $\mathrm{pVO}_{2} \leq 12 \mathrm{BB} / 14 \mathrm{ml} / \mathrm{kg} / \mathrm{min}$ and 0.05 ppy in those with $\mathrm{pVO}_{2}>12 \mathrm{BB} / 14 \mathrm{ml} / \mathrm{kg} / \mathrm{min}(P=0.002)$.

\section{DISCUSSION}

The current study is the first to demonstrate that lower values of $\mathrm{pVO}_{2}$ are highly predictive of death (Central Illustration) and HF hospitalizations in LVAD recipients. It is further shown that another measure which can be obtained during $\mathrm{CPET}, \mathrm{VE} / \mathrm{VCO}_{2}$ slope, predicts survival in LVAD recipients as is the case for patients with HF not supported by MCS.

The prognostic power of $\mathrm{pVO}_{2}$ in heart failure patients considered for transplantation was demonstrated in the landmark study from 1991 by Mancini et al (11). Since then, several studies established the prognostic value of $\mathrm{pVO}_{2}$ in advanced heart failure populations $(17,18)$. The present results confirm that these findings are valid in recipients of durable LVADs, as $\mathrm{pVO}_{2}$ provided robust prognostic information in stable, ambulatory LVAD recipients, both in the early and late phases ( $>6$ months) after implantation. The cutoff of 12 (if patients were taking $\mathrm{BB}$ ) or 14 (if not taking $\mathrm{BB}$ ) $\mathrm{ml} /$ $\mathrm{kg} / \mathrm{min}$ is widely used in patients with advanced HF not supported by MCS to determine timing of transplantation (10), and we demonstrate that this threshold can also be applied in LVAD recipients. Interestingly, $\mathrm{pVO}_{2}$ also predicted the risk of $\mathrm{HF}$ hospitalizations which is especially relevant for the growing population of DT patients (19).

Peak oxygen uptake is a reproducible, objective and dynamic parameter which assesses the cardiovascular reserve and peripheral adaptions to reduced cardiac output (according to Fick's principle) and as a direct consequence is highly predictive of outcome in HF $(11,20,21)$. In LVAD recipients, total cardiac output (CO) is determined by blood flow through the pump and the native CO (through the aortic valve), the latter contributing in particular during exercise. Recently, Moss et al (22) presented exercise hemodynamic data and simultaneous $\mathrm{VO}_{2}$ measurements 
B

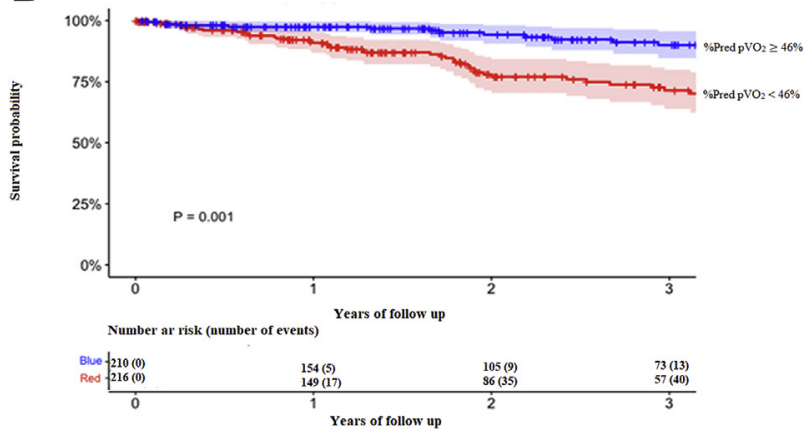

C

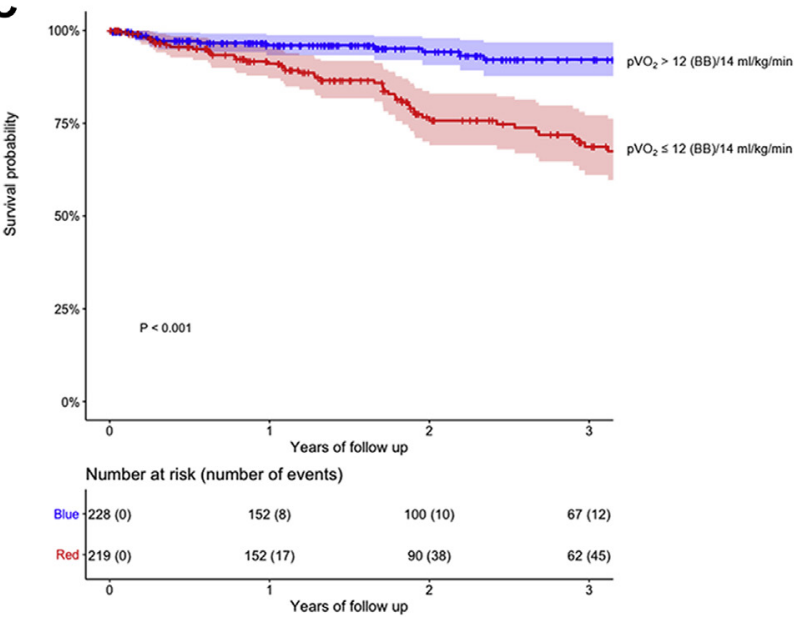

in $45 \mathrm{LVAD}$ recipients. The authors confirmed that the main determinant of $\mathrm{pVO}_{2}$ was patients' ability to increase total $\mathrm{CO}$ and, second, the capacity to extract oxygen peripherally (ie, arterial venous oxygen difference). Several factors contribute to the low CO and, consequently, $\mathrm{pVO}_{2}$ observed in LVAD recipients. Although comorbidity and pump speed settings play a role (23), main determinants are LV function (ie, myocardial recovery) (24), aortic and mitral valve disease $(25,26)$, and chronotropic incompetence $(27,28)$. Although RV function has been shown to influence $\mathrm{pVO}_{2}$ in some (29) but not all (27) studies, RV failure is clearly associated with increased risk of readmission $(30,31)$ and poorer overall outcome in LVAD recipients $(32,33)$. Taken together, these findings point to underlying mechanisms relating to incompletely treated heart failure as the main cause for low $\mathrm{pVO}_{2}$ in LVAD recipients, which in turn, is consistent with the striking similarity between the $\mathrm{pVO}_{2}$, survival relationship observed in LVAD recipients in the current study, and that reported for patients with HF not supported by MCS.
Several studies (7) have reported $\mathrm{pVO}_{2}$ in LVAD recipients with values ranging from 9.9 (34) to 20.3 (35) $\mathrm{ml} / \mathrm{kg} / \mathrm{min}$ in populations consisting mostly of middle-aged patients (ages range from 35 to 63 years old) (35-37). Although age ranges in earlier reports are comparable to those found in the PRO-VAD study, $\mathrm{pVO}_{2}$ values in the current investigation ranged from 4.0 to $35.6 \mathrm{ml} / \mathrm{kg} / \mathrm{min}$. This indicates that the examined population is quite heterogeneous, including patients implanted with DT, BTT, and bridge-todecision indications. We included all 3 categories for several reasons. First, a considerable number of patients designated DT regain candidacy after LVAD implantation and undergo transplantation, whereas conversely, some patients implanted with a BTT strategy develop contraindications to transplantation. Second, recent data suggest that an important difference in outcome in DT and BTT or bridge-to-candidacy patients do not exist, which, together with the crossover described, has led to the suggestion of abandoning the designations BTT and DT entirely (38). In the current study, it is further 


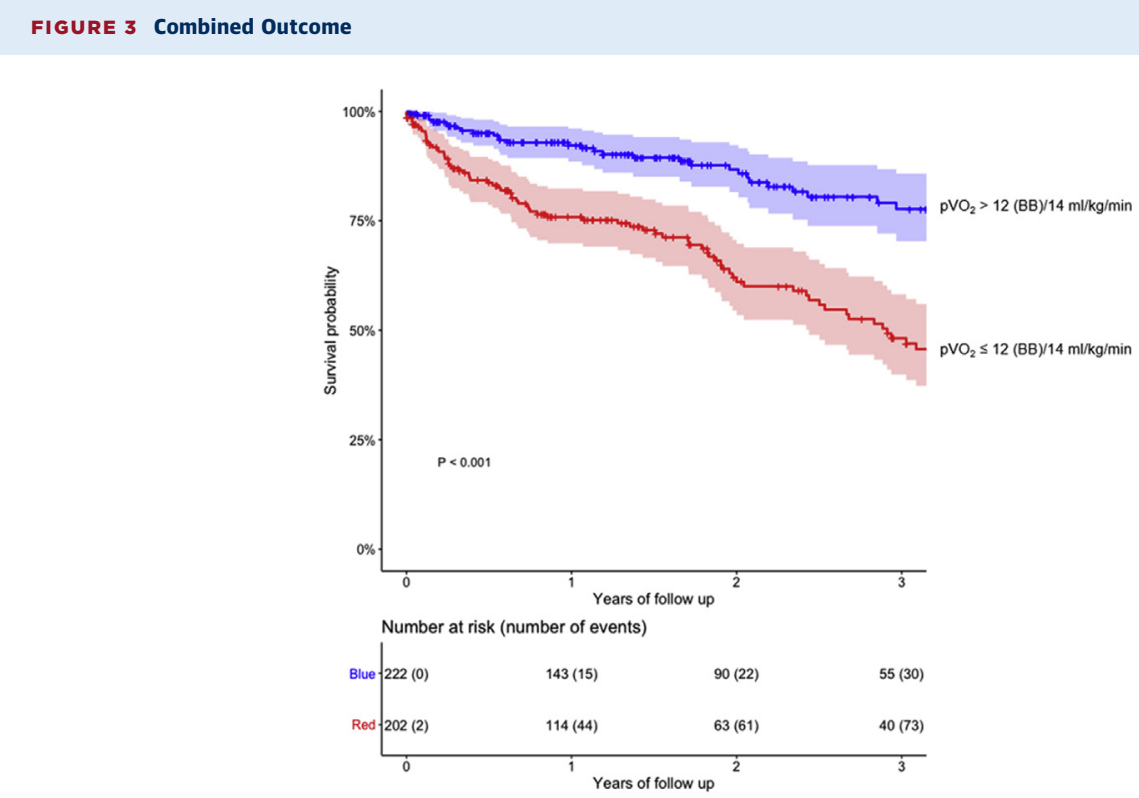

Combined outcome (death or heart failure hospitalization) predicted by peak oxygen uptake $\left(\mathrm{pVO}_{2}\right)$ in $\mathrm{ml} / \mathrm{kg} / \mathrm{min}$ using the ISHLT cutoffs for evaluating transplant listing. ISHLT = International Society for Heart and Lung Transplantation.

hemodynamic assessment. Additionally, the CPET study may serve as an excellent tool in determining the need for additional physical training in these patients.

STUDY LIMITATIONS. Although the current study is strengthened by its multicenter design and the large sample size, some limitations remain. The data were collected retrospectively, leading to missing information of some datapoints for some patients, especially regarding test protocols, LVAD settings, and echocardiographic findings, etc. Information for cause of death was not available. The study did not rely on enrollment of consecutive patients and the results cannot be extrapolated to patients with characteristics different from those of our study population. Patients not able to perform exercise tests were not included, which may be a major prognosticator limiting the extrapolation of the results. Furthermore, the authors did not have access to information regarding adverse events or quality of life in this study. The patients did not undergo CPET at a prespecified timepoint and exercise protocols differed between centers. Furthermore, the patients received implants from a wide range of durable LVADs, thus reflecting a large proportion of the current LVAD landscape. This, however, is not different from advanced patients with HF not supported with MCS who may undergo CPET at any point in their disease journey, typically to assess if transplant listing is relevant. Reassuringly, the prognostic power of $\mathrm{pVO}_{2}$ in the current study did not depend on the timing after LVAD implantation. Only $6 \%$ of patients were tested on a treadmill, and as such the findings of the current study should be applied with caution to patients subjected to this test modality.

\section{CONCLUSIONS}

In this large multicenter study, the authors demonstrated that the cardiovascular reserve expressed as peak oxygen uptake is of clear prognostic value in LVAD recipients, comparable to heart failure patients not supported by MCS. Risk stratification of patients with LVAD awaiting heart transplant by $\mathrm{pVO}_{2}$ and VE/ $\mathrm{VCO}_{2}$ slope should be considered. Furthermore, since $\mathrm{pVO}_{2}$ predicts $\mathrm{HF}$ hospitalizations after LVAD implantation, routine postimplant measurement of oxygen consumption and aggressive therapy in patients with poor results may be helpful to improve outcomes in this population.

ACKNOWLEDGMENTS The authors thank the contributing centers, including every clinician and patient, without whom this project would not have been possible. 


\section{CENTRAL ILLUSTRATION Prognostic Impact of Peak Oxygen Uptake}

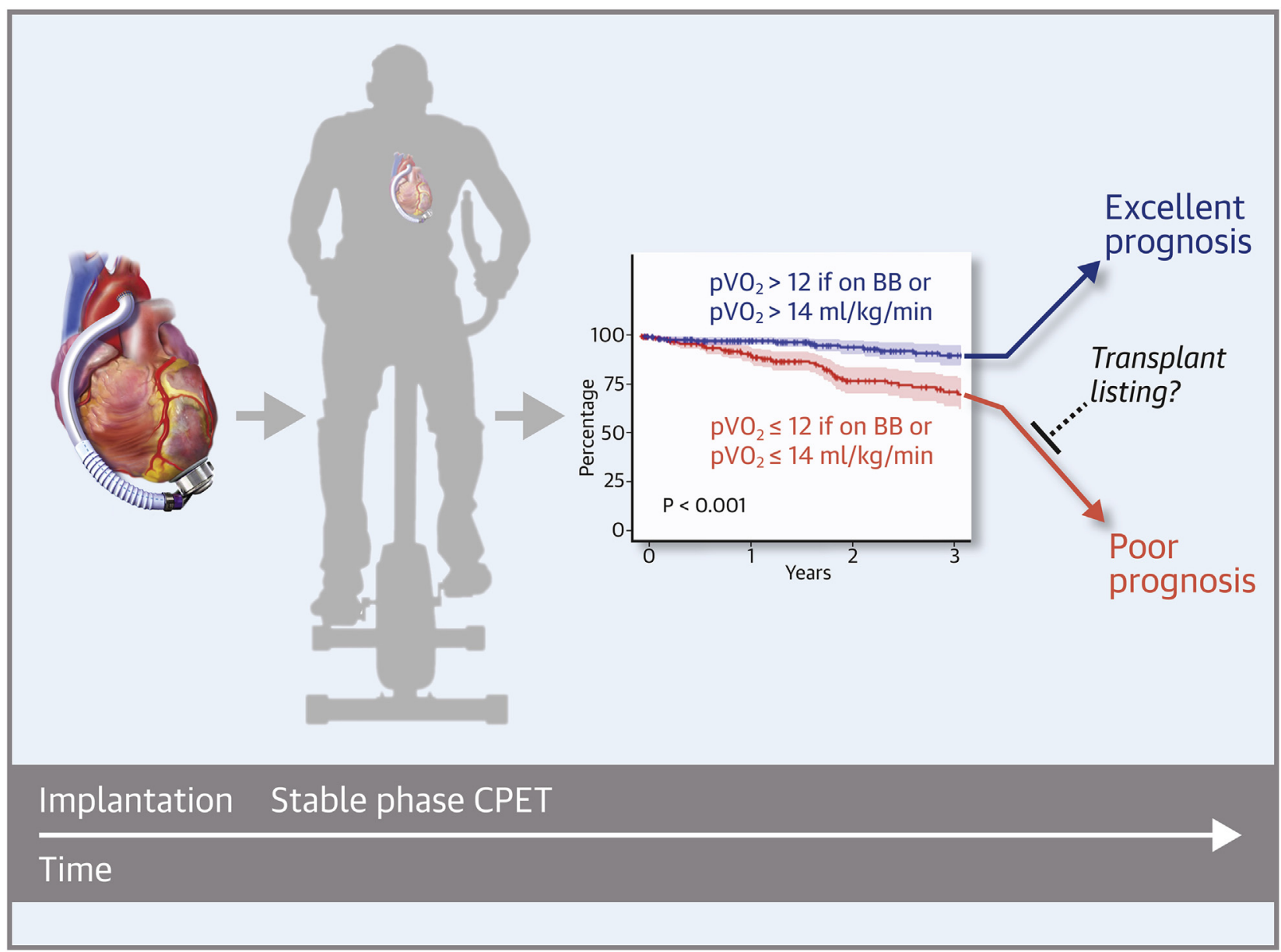

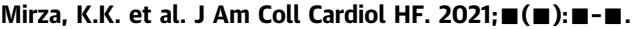

Low peak oxygen uptake ( $\mathrm{pVO}_{2}<12 \mathrm{ml} / \mathrm{kg} / \mathrm{min}$ (if treated with beta-blockers) or $<14 \mathrm{ml} / \mathrm{kg} / \mathrm{min}$ (no beta-blocker) predicts mortality in stable recipients of a continuous-flow LVAD. The median time from LVAD implantation to the exercise test was approximately 6 months. The findings are remarkably similar to those observed for HF patients without LVAD support, where the same cutoff values for $\mathrm{pVO}_{2}$ are used to inform decisions for listing heart transplantation.

\section{FUNDING SUPPORT AND AUTHOR DISCLOSURES}

Dr Mirza received Danish funding from Skibsreder Per Henriksen, R og hustrus Fond grant 10403/53 and Jascha Fonden grants 6186 and 7724. Dr Gustafsson is supported by research grant NNF200Co06056 from the Novo Nordisk Foundation. Dr Brahmbhatt has received travel support from Abbott, Biotronik; and has received honoraria, travel support, and research funding from Boston Scientific (all outside the submitted work). Dr Hsu has received U.S. National Institutes of Health funding (grant K23-HL146889) (all outside the submitted work). Dr MacGowan is supported by EU Horizon 2020 and United Kingdom National Institute of Health Research. Dr Jakovljevic is supported by EU Horizon 2020 Research and Innovation Programme under grant agreements 777204 and 952603. Dr Gustafsson has received personal fees from Abbott, Carmat, Pfizer, Novartis,
Orion Pharma, Boehringer Ingelheim, Pharmacosmos, Amgen, Bayer, and AstraZeneca; and is a consultant for Abbott, Carmat, Pfizer, Novartis, Orion Pharma, Boehringer-Ingelheim, Pharmacosmos, Amgen, Bayer and AstraZeneca (all outside the submitted work). Dr Russell is a consultant for Medtronic and Abbott (all outside the submitted work). Dr Jorde is a consultant for Abbott (all outside the submitted work). All other authors have reported that they have no relationships relevant to the contents of this paper to disclose.

ADDRESS FOR CORRESPONDENCE: Dr Kiran Kafila Mirza, Department of Cardiology, Copenhagen University Hospital-Rigshospitalet, Blegdamsvej no. 9, DK-2100 Copenhagen, Denmark. E-mail: RNZ440@ sund.ku.dk. Twitter: @KiranKMirza, @FinnGustafsson. 
COMPETENCY IN MEDICAL KNOWLEDGE: Cardiovascular reserve expressed as peak oxygen uptake is of clear prognostic value in LVAD recipients, comparable to that in heart failure patients not supported by MCS. As $\mathrm{pVO}_{2}$ predicts $\mathrm{HF}$ hospitalizations after LVAD implantation, routine post-implant measurement of oxygen consumption and aggressive therapy in patients with poor results might be helpful to improve outcomes LVAD recipients. Risk stratification of patients with LVAD supporting heart transplant listing decisions by $\mathrm{pVO}_{2}$ and $\mathrm{VE} / \mathrm{VCO}_{2}$ slope should be considered.

TRANSLATIONAL OUTLOOK: In LVAD recipients the use of peak oxygen uptake can guide clinicians to select patients with increased risk of death and heart failure hospitalizations. Risk stratification of patients with LVAD supporting heart transplant listing decisions by $\mathrm{pVO}_{2}$ and $\mathrm{VE} / \mathrm{VCO}_{2}$ slope should be considered.

\section{REFERENCES}

1. Chatterjee A, Feldmann $C$, Hanke JS, et al. The momentum of HeartMate 3: A novel active magnetically levitated centrifugal left ventricular assist device (LVAD). J Thorac Dis. 2018;10:S1790-S1793.

2. Mehra MR, Goldstein DJ, Uriel $N$, et al. Twoyear outcomes with a magnetically levitated cardiac pump in heart failure. $N$ Engl J Med. 2018;378:1386-1395.

3. Gustafsson F, Shaw S, Lavee J, et al. Six-month outcomes after treatment of advanced heart failure with a full magnetically levitated continuous flow left ventricular assist device: Report from the ELEVATE registry. Eur Heart J. 2018;39:34543460.

4. Theochari CA, Michalopoulos G, Oikonomou EK, et al. Heart transplantation versus left ventricular assist devices as destination therapy or bridge to transplantation for 1-year mortality: a systematic review and meta-analysis. Ann Cardiothorac Surg. 2018;7:3-11.

5. Cowger $J A$, Naka $Y$, Aaronson $K D$, et al, Quality of life and functional capacity outcomes in the MOMENTUM 3 trial at 6 months: a call for new metrics for left ventricular assist device patients. J Heart Lung Transplant. 2018;37: 15-24.

6. Rogers JG, Aaronson KD, Boyle AJ, et al. Continuous flow left ventricular assist device improves functional capacity and quality of life of advanced heart failure patients. J Am Coll Cardiol. 2010;55:1826-1834.

7. Mirza KK, Gustafsson F. Determinants of functional capacity and quality of life after implantation of a durable left ventricular assist device. Card Fail Rev. 2020;6:e29.

8. Zimpfer D, Gustafsson F, Potapov E, et al. Twoyear outcome after implantation of a full magnetically levitated left ventricular assist device: results from the ELEVATE Registry. Eur Heart J. 2020;41:3801-3809.

9. Rogers JG. Changes in United States heart allocation: a community energized to improve policy. J Thorac Cardiovasc Surg. 2016;152:14841486.
10. Mehra MR, Canter CE, Hannan MM, et al. The 2016 International Society for Heart Lung Transplantation listing criteria for heart transplantation a 10-year update. J Heart Lung Transplant 2016;35:1-23.

11. Mancini DM, Eisen $H$, Kussmaul W, Mull $R$ Edmonds LH, Wilson JR. Value of peak exercise oxygen consumption for optimal timing of cardiac transplantation in ambulatory patients with heart failure. Circulation. 1991;83:778-786.

12. MacGowan GA, Janosko $K$, Cecchetti $A$ Murali S. Exercise-related ventilatory abnormalities and survival in congestive heart failure. Am J Cardiol. 1997:79:1264-1266.

13. Hansen JE, Sue DY, Wasserman K. Predicted values for clinical exercise testing. Am Rev Respir Dis. 1984;129:S49-S55.

14. Arena $R$, Myers J, Abella J, et al. Determining the preferred percent-predicted equation for peak oxygen consumption in patients with heart failure. Circ Heart Fail. 2009;2:113-120.

15. Tabet JY, Beauvais F, Thabut G, Tartière JM, Logeart D, Cohen-Solal A. A critical appraisal of the prognostic value of the $\mathrm{VE} / \mathrm{VCo} 2$ slope in chronic heart failure. Eur J Cardiovasc Prev Reha bil. 2003;10:267-272.

16. Corrà U, Piepoli MF, Adamopoulos $S$, et al. Cardiopulmonary exercise testing in systolic hear failure in 2014: the evolving prognostic role a position paper from the committee on exercise physiology and training of the heart failure association of the ESC. Eur J Heart Fail. 2014;16:929941.

17. Davies LC, Francis DP, Piepoli M, Scott AC, Ponikowski $\mathrm{P}$, Coats AJS. Chronic heart failure in the elderly: Value of cardiopulmonary exercise testing in risk stratification. Heart. 2000;83:147151.

18. Cohenl-Solal A, Caviezel B. Cardiopulmonary exercise testing in chronic heart failure. Heart Fail. 1994; 10:46-57.

19. Ponikowski P, Voors AA, Anker SD, et al. 2016 ESC Guidelines for the diagnosis and treatment of acute and chronic heart failure. Eur Heart $J$. 2016;37:2129-2200.

20. Jessup Likoff M, Chandler SL, Kay HR. Clinical determinants of mortality in chronic congestive heart failure secondary to idiopathic dilated or to ischemic cardiomyopathy. Am J Cardiol. 1987;59: 634-638.

21. Szlachcic J, Masse BM, Kramer BL, Topic $N$, Tubau J. Correlates and prognostic implication of exercise capacity in chronic congestive heart failure. Am J Cardiol. 1985;55:1037-1042.

22. Moss $N$, Rakita V, Lala $A$, et al. Hemodynamic response to exercise in patients supported by continuous flow left ventricular assist devices. J Am Coll Cardiol HF. 2020;8:291-301.

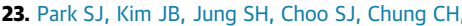
Lee JW. Outcomes of extracorporeal life support for low cardiac output syndrome after major cardiac surgery. J Thorac Cardiovasc Surg. 2014;147: 283-289.

24. Birks EJ, Drakos SG, Patel SR, et al. Prospective multicenter study of myocardial recovery using left ventricular assist devices (RESTAGE-HF [Remission from Stage D Heart Failure]): mediumterm and primary end point results. Circulation. 2020:142:2016-2028.

25. McKellar SH, Deo S, Daly RC, et al. Durability of central aortic valve closure in patients with continuous flow left ventricular assist devices. J Thorac Cardiovasc Surg. 2014;147:344-348.

26. Imamura $T$, Kinugawa $K$, Nitta $D$, et al. Opening of aortic valve during exercise is key to preventing development of aortic insufficiency during ventricular assist device treatment. ASAIOJ. 2015;61:514-519.

27. Mirza $K K$, Jung $M H$, Sigvardsen $P E$, et al. Computed tomography-estimated right ventricular function and exercise capacity in patients with continuous-flow left ventricular assist devices. ASAIOJ. 2020;66:8-16.

28. Alvarez Villela $M$, Guerrero-Miranda $C Y$, Chinnadurai T, Patel SR, Jorde UP. Rate response pacing in left ventricular assist device patients. ASAIOJ. 2020;66:e29-e30. 
29. Bouzas-Cruz N, Koshy A, GonzalezFernandez $\mathrm{O}$, et al. Markers of right ventricular dysfunction predict maximal exercise capacity after left ventricular assist device implantation. ASAIO J. 2020 [ahead of print].

30. Hasin $T$, Marmor $Y$, Kremers $W$, et al. Readmissions after implantation of axial flow left ventricular assist device. J Am Coll Cardiol. 2013;61: 153-163.

31. Smedira NG, Hoercher KJ, Lima B, et al. Unplanned hospital readmissions after heartmate ॥ implantation. Frequency, risk factors, and impact on resource use and survival. J Am Coll Cardiol HF. 2013;1:31-39.

32. Takeda $\mathrm{K}$, Takayama $\mathrm{H}$, Colombo $\mathrm{PC}$, et al. Incidence and clinical significance of late right heart failure during continuous-flow left ventricular assist device support. J Heart Lung Transplant. 2015;34:1024-1032.

33. Goldstein DJ, Meyns B, Xie R, et al. Third annual report from the ISHLT mechanically assis- ted circulatory support registry: a comparison of centrifugal and axial continuous-flow left ventricular assist devices. J Heart Lung Transplant. 2019;38:352-363.

34. Gross C, Marko C, Mikl J, et al. LVAD Pump flow does not adequately increase with exercise. Artif Organs. 2019;43:222-228.

35. Noor MR, Bowles $C$, Banner NR. Relationship between pump speed and exercise capacity during HeartMate II left ventricular assist device support: Influence of residual left ventricular function. Eur J Heart Fail. 2012;14:613-620.

36. Rosenbaum AN, Dunlay $S M$, Pereira NL, et al. Determinants of improvement in cardiopulmonary exercise testing after left ventricular assist device implantation. ASAIOJ. 2018;64:610-615.

37. Schmidt $\quad T$, Bjarnason-Wehrens $\quad B$, Mommertz S, et al. Development of exerciserelated values in heart failure patients supported with a left ventricular assist device. Int J Artif Organs. 2019;42:201-206.
38. Goldstein DJ, Naka Y, Horstmanshof D, et al. Association of clinical outcomes with left ventricular assist device use by bridge to transplant or destination therapy intent: the multicenter study of MagLev technology in patients undergoing mechanical circulatory support therapy with HeartMate 3. JAMA Cardiol. 2020;5:411-419.

39. Uriel N, Burkhoff D, Rich JD, et al. Impact of hemodynamic ramp test-guided HVAD speed and medication adjustments on clinical outcomes: the RAMP-IT-UP multicenter study. Circ Hear Fail. 2019;12:12-22.

KEY WORDS exercise, maximum oxygen uptake, outcome, prognosis, survival

APPENDIX For supplemental tables and figures, please see the supplemental version of this paper. 